\title{
MS27-P07 | UNIQUE PROPERTIES OF GEOINSPIRED NANOTUBES AS WATER NANOCONTAINER
}

MONET, Geoffrey (Université Paris Saclay, Orsay, FRA); CHAl, Ziwei (Beijing Computational Science Research Centre, Beijing, CHN); PAINEAU, Erwan (CNRS, Orsay, FRA); LIU, Li-Min (Beijing Computational Science Research Centre, Beijing, CHN); TEOBALDI, Gilberto (Daresbury laboratory, Daresbury, GBR); ROLS, Stéphane (Institut Laue-Langevin, Grenoble, FRA); LAUNOIS, Pascale (CNRS, Orsay, FRA)

Clay-like imogolite nanotubes (INT) are ideal platforms for studying the novel properties of confined molecules. Compared to the well-known carbon nanotubes (CNT), they own well defined diameter. Moreover, the molecular affinity of their inner cavity is tunable. Such properties confer to these tubular structures a wide range of potential applications, from filtration to depollution of water.

X-ray and inelastic neutron scattering techniques are used here, in combination with simulations, to investigate structural and dynamical properties at the nanoscale. We performed inelastic neutron scattering (INS) experiments to study hydrated geo-inspired alumino-germanate imogolite nanotubes (Ge-INT) [1], whose structure is first determined thanks to X-ray scattering [1,2]. INS experiments coupled to ab-initio molecular dynamics simulations reveal a unique structure of the water layer adsorbed inside Ge-INT. To the best of our knowledge, it differs from that of any kind of two-dimensional water. We show that the dynamics of INT and water are strongly interrelated, so that hydrated Ge-INT can be considered as a different system from dry Ge-INT. A well-defined translation vibrational mode of water molecules with respect to the nanotube wall is also observed. Finally, the evolution of mean square displacements of water molecules as a function of temperature is found to differ markedly in Ge-INT and in CNT, in which water slips without friction, which is attributed to the peculiar bonding of water molecules with germanol groups.

[1] G. Monet et al., in preparation

[2] G. Monet et al., Nature Comm., 9, 2033 (2018) 